\title{
Experimental research and numerical modelling of the movement of explosive and deflagrable clouds
}

\author{
Y. Zheng, F. Wang, P. Li, X. Li, W. Liu, G. Wu \& H. Zhang \\ Research Institute of Chemical Defense, China
}

\begin{abstract}
In order to achieve a movement rule for contaminative clouds by explosion and deflagration, field experiments were carried out during the summer of 2010 in which the process of the fireball and movement of the clouds were recorded respectively using high speed CCD recorders, video cameras and infrared photography. Based on the assumption that the main effects on the ascending movement of clouds are gravity and buoyancy, we provide a numerical model for the movement of the clouds that considers the meteorological conditions. The modelling results are reasonable compared to the data obtained from the experiment, which can serve as a reference to the related fields.

Keywords: contaminative clouds, explosion, deflagration, field experiments, numerical model.
\end{abstract}

\section{Introduction}

Contaminative events, especially a "dirty bomb" or other mephitic vapour explosions, have received a great deal of attention. In order to study the movement of the clouds by explosion and deflagration, experiments and numerical modelling methods have been put forward by a number of scientists. Generally speaking, two kinds of experimental methods are used, one is performed in the laboratory and another is in the field.

After the similar movement theory of the instantaneous heat sources was put forward by Taylor [1], Morton et al. [2] performed an experiment on the different densities of liquid in the box gutter to model the movement of the thermal energy in the atmosphere. Zaslavskii and Sconikov [3] designed a method of igniting the 
bubbles of $\mathrm{He}, \mathrm{N}_{2}$ and $\mathrm{O}_{2}$ mixture to produce the buoyancy vortex rings. Zheng [4] used the instantaneous heat sources to study the movement of the buoyant vortex rings by igniting the bubbles of $\mathrm{C}_{2} \mathrm{H}_{4}$ and $\mathrm{O}_{2}$ mixture to produce combustion fireballs and smokes in laboratory. Thielen and Schrodl [5] used two cameras to record the movement of the explosive cloud and compared test results with the HOTSPOT health physics codes. The experiment indicated that the approach was reasonable.

On the other hand, the development of numerical calculation have provided the support for the study of the explosive cloud. Makhviladze et al. [6] developed a model for simulating the pollution of the explosion. Krispin and Collins [7] and Brown et al. [8] used projection method to calculate the development of the low density bubble. They have provided reasonable results of the explosive bubble without considering the meteorological condition and had not given comparison to the experiment data.

In this paper, we performed the experiments in the field and numerical modelling to study the mechanism of the ascending of the explosive and deflagration cloud. The experiments have been performed using two kinds of video recorders, the high speed CCD recorders and the video cameras, to record the process of the fireball and the movement of the cloud respectively. Based on the assumption that the main effects on the clouds ascending movement are gravity and buoyancy, we provided numerical model on the movement of the thermal considering the meteorological condition.

\section{Experimental design}

The experiments were performed from July 17 to August 4, 2010. We chose a training area as the test site at Beijing northwest suburb. Figure 1 shows the experimental field. Figure 2 shows the experimental devices. The exploders are placed 1.5 meters above the ground. Two high speed CCD recorders of MotionXtra HG-LE are laid from a distant about 100m, which are used to record the change of the explosion fireball. At the same time, two video cameras of SONY are placed in another two different directions about $200 \mathrm{~m}$ used to record the movement of the explosive cloud.

To consider the influence of meteorological conditions, a meteorological measuring tower is set near the exploder site about 400 meters to provide meteorological observation, which had 12 meters high and could capture 3 levels parameters such as pressure, temperature, density and wind, on $4 \mathrm{~m}, 7.5 \mathrm{~m}$ and $11 \mathrm{~m}$ respectively.

\subsection{Explosive experiments results}

We carried 23 times explosive experiments altogether, the explosive material were RDX and TNT, and the equivalent TNT masses from $0.1 \mathrm{~kg}$ to $10.0 \mathrm{~kg}$. Table 1 contains the matrix of the explosive experiments. 


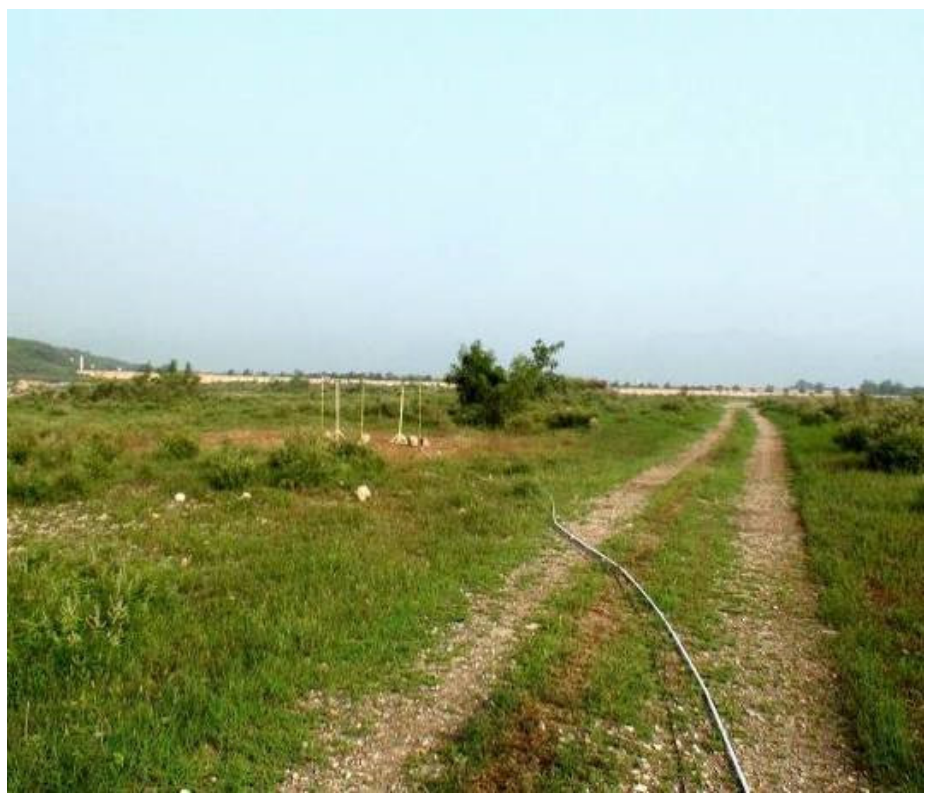

Figure 1: The experimental field.

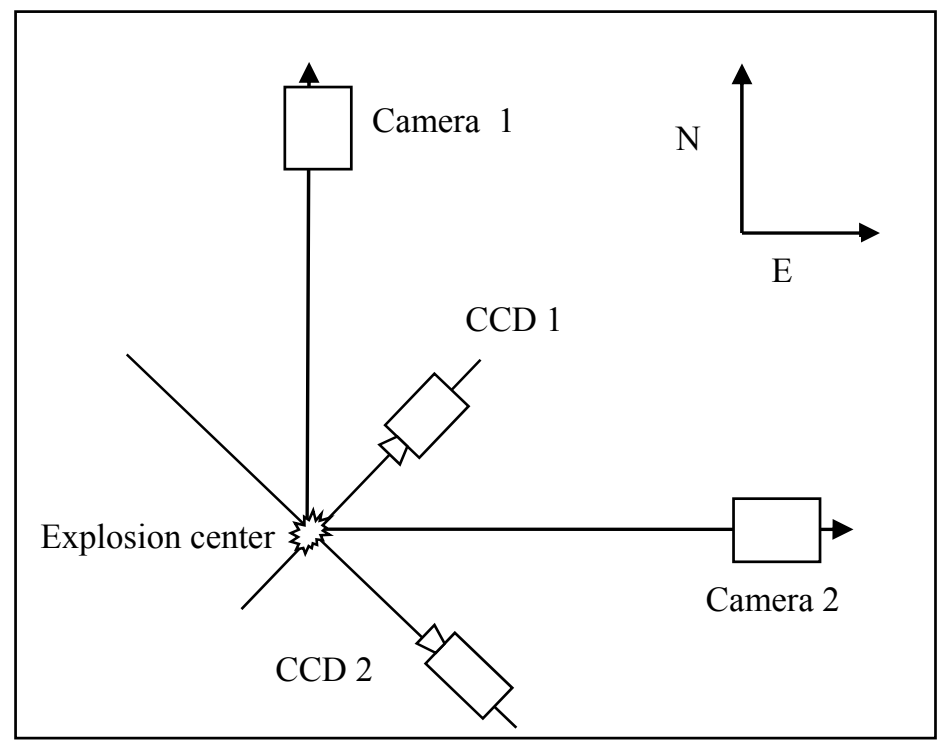

Figure 2: Sketch of the explosion experiment. 
Table 1: Explosive experiments matrix.

\begin{tabular}{|c|c|c|c|c|}
\hline Test & $\begin{array}{c}\text { Explosive } \\
\text { Material }\end{array}$ & $\begin{array}{c}\text { Equivalent TNT } \\
\text { mass } \\
/ \mathrm{kg}\end{array}$ & $\begin{array}{l}\text { Recording } \\
\text { altitude } \\
/ \mathrm{m}\end{array}$ & $\begin{array}{c}\text { Recording } \\
\text { time } \\
/ \mathrm{s}\end{array}$ \\
\hline BZ1 & RDX & 0.1 & 33.6 & 35 \\
\hline BZ2 & RDX & 0.1 & 21.7 & 15 \\
\hline BZ3 & RDX & 0.1 & 17.9 & 40 \\
\hline BZ4 & $\mathrm{RDX}$ & 0.1 & 11.8 & 17 \\
\hline BZ5 & TNT & 0.1 & & \\
\hline BZ6 & TNT & 0.1 & 30.9 & 54 \\
\hline BZ7 & TNT & 0.1 & 33.9 & 60 \\
\hline BZ8 & TNT & 0.1 & 19.2 & 27 \\
\hline BZ9 & TNT & 0.1 & 14.8 & 8 \\
\hline BZ10 & TNT & 0.1 & 11.9 & 8 \\
\hline BZ11 & RDX & 0.5 & 26.9 & 71 \\
\hline BZ12 & TNT & 0.5 & 27.37 & 21 \\
\hline BZ13 & RDX & 1.0 & 25.4 & 34 \\
\hline BZ14 & TNT & 1.0 & 23.2 & 45 \\
\hline BZ15 & TNT & 1.0 & 30.7 & 14 \\
\hline BZ16 & TNT & 1.0 & 30.89 & 16 \\
\hline BZ17 & TNT & 1.0 & 29.02 & 21 \\
\hline BZ18 & TNT & 1.0 & 48.3 & 30 \\
\hline BZ19 & TNT & 1.0 & 46.7 & 34 \\
\hline BZ20 & $\mathrm{RDX}$ & 5.0 & 40.5 & 33 \\
\hline BZ21 & RDX & 5.0 & 38.4 & 34 \\
\hline BZ22 & RDX & 5.0 & 34.8 & 35 \\
\hline BZ23 & RDX & 10.0 & 61.3 & 35 \\
\hline
\end{tabular}

"Recording altitude" and "Recording time" represent the maximal recording top altitude and the maximal recording time of the cloud respectively.

Figure 3 shows a picture of explosion clouds from two observed directions at different times of test BZ7. 


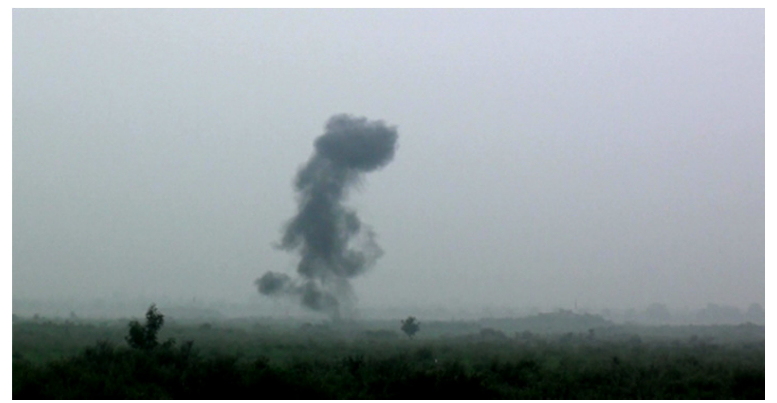

(a) From the direction of camera $1(\mathrm{t}=20 \mathrm{~s})$

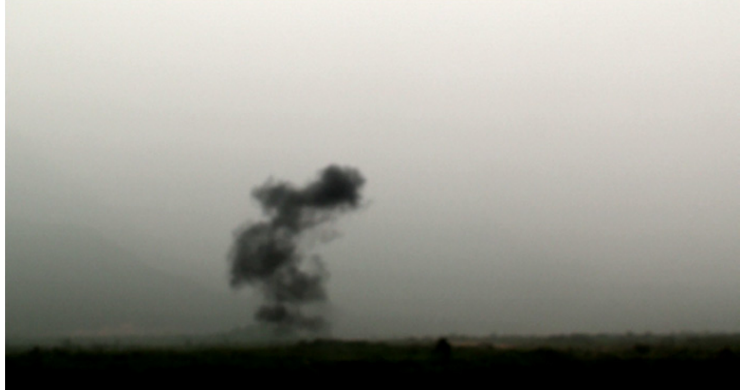

(b) From the direction of camera $2(t=20 \mathrm{~s})$

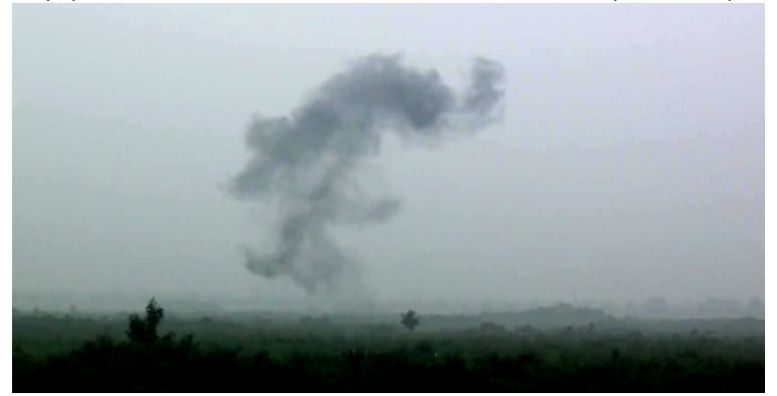

(c) From the direction of camera $1(t=35 \mathrm{~s})$

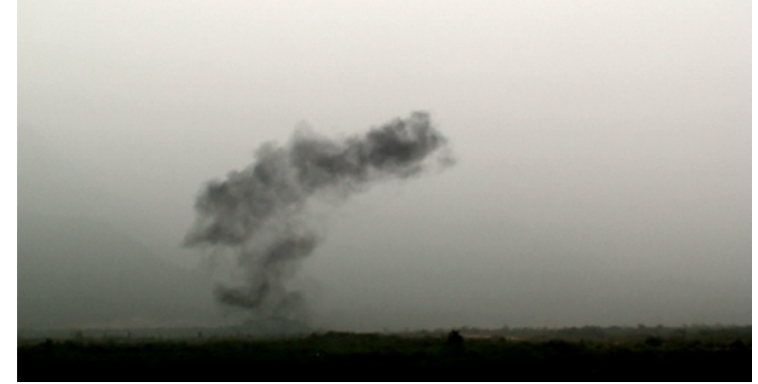

(d) From the direction of camera $2(t=35 \mathrm{~s})$

Figure 3: Explosion clouds from two observed directions at different times of test BZ7. 


\subsection{Deflagrable experiment results}

Several deflagrable experiments have been carried out through during the same time. The recording method was the same as the explosive experiment. The difference between explosion and deflagration tests is in the producing of the cloud. Deflagration clouds are created by burning of the fuel using the equipment of the deflagrator but no chemical explosion taking place. When the detonator is ignited, the liquid fuel can be jetted into the air and ignited instantly. At the same time, we adopt the infrared photography of DALI DM60 to obtain the temperature of the fireball during the deflagrable experiments. Table 2 contains the matrix of the deflagrable experiments.

Table 2: Deflagrable experiments matrix.

\begin{tabular}{|c|c|c|c|c|}
\hline Test & Fuel & Volume & $\begin{array}{c}\text { Recording } \\
\text { radius } \\
/ \mathrm{m}\end{array}$ & $\begin{array}{c}\text { Recording } \\
\text { altitude } \\
/ \mathrm{m}\end{array}$ \\
\hline BR1 & gasoline & 4L & & \\
\hline BR2 & gasoline & 4L & 9.4 & 13.04 \\
\hline BR3 & gasoline & 4L & & \\
\hline BR4 & gasoline & 4L & 17.7 & 38 \\
\hline BR5 & gasoline & 4L & 19.6 & 40.9 \\
\hline BR6 & gasoline & 4L & 9.9 & 32.9 \\
\hline BR7 & gasoline & 4L & 15.4 & 33.2 \\
\hline
\end{tabular}

"Volume" represents the volume of the fuel, "Recording radius" and "Recording altitude" represent the maximal recording radius and altitude of the cloud respectively during the recording time.

Figure 4 shows the states of the fireball at different times from high speed CCD recorder of BR2 test, the frequency of the CCD recording was 1000 frame/s.

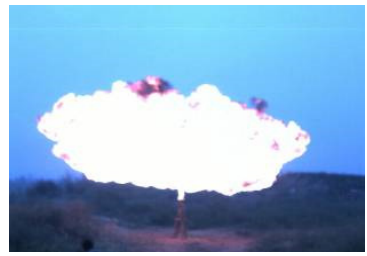

(a) $\mathrm{t}=100 \mathrm{~ms}$

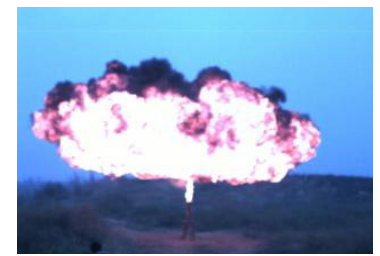

(b) $\mathrm{t}=300 \mathrm{~ms}$

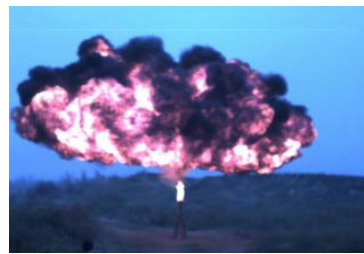

(c) $\mathrm{t}=500 \mathrm{~ms}$

Figure 4: Fireballs at different times of deflagrable test BR2.

Figure 5 shows the picture of the temperature increment of fireball during the deflagrable test BR4 and BR6. 


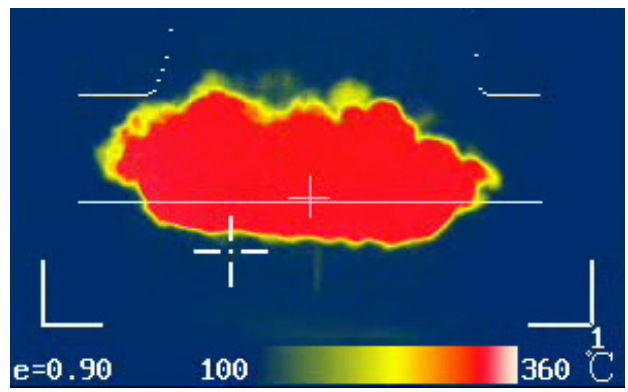

(a) BR4 experiment

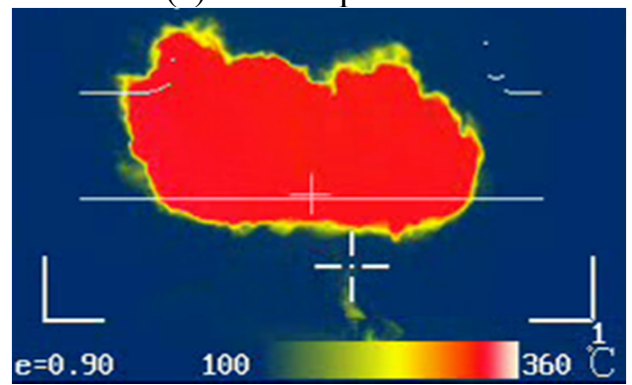

(b) BR6 experiment

Figure 5: Temperature increment of the deflagrable experiment.

\section{Numerical model}

Suppose that the main mechanism of the explosive bubble rising is the buoyancy. Since the top altitude of the cloud is about 50 meters from the experiments, we assume that the calculated domain $(60 \mathrm{~m} \times 60 \mathrm{~m} \times 60 \mathrm{~m})$ fill with incompressible gas, a spherical thermal with radius of $\mathrm{R}$ fill with higher temperature air and locate at $1.5 \mathrm{~m}$ above the domain bottom. The equation can be written as the following:

$$
\begin{gathered}
\frac{\partial u^{\prime}}{\partial t}+\mathbf{U} \bullet \nabla u^{\prime}=-\frac{1}{\rho} \frac{\partial p^{\prime}}{\partial x} \\
\frac{\partial v^{\prime}}{\partial t}+\mathbf{U} \bullet \nabla v^{\prime}=-\frac{1}{=} \frac{\partial p^{\prime}}{\partial y} \\
\frac{\partial w^{\prime}}{\partial t}+\mathbf{U} \bullet \nabla w^{\prime}=-\frac{1}{\rho} \frac{\partial p^{\prime}}{\partial z}+g \frac{\theta^{\prime}}{\bar{\theta}} \\
\frac{\partial \theta^{\prime}}{\partial t}+\mathbf{U} \bullet \nabla \theta^{\prime}+\frac{N^{2} \bar{\theta}}{g} w=0
\end{gathered}
$$




$$
\frac{\partial u^{\prime}}{\partial x}+\frac{\partial v^{\prime}}{\partial y}+\frac{\partial w^{\prime}}{\partial z}=0
$$

where $u^{\prime}, v^{\prime}, w^{\prime}, \theta^{\prime}, p^{\prime}$ represent the disturbance of three directions velocity, potential temperature and pressure. $\mathrm{U}, \bar{\rho}, \bar{\theta}$ represent the mean velocity, mean density and mean potential temperature. $N$ represents the Brunt-Vaisala frequency.

\subsection{Potential temperature increment of the explosive experiment}

The potential temperature increment can be calculated by the equation of the following:

$$
Q=C_{v} \bar{\rho} V_{0} \Delta \theta
$$

where $Q$ is the energy of the explosion, and we assume that about $1 / 3$ of the explosive energy were transformed to the heat. $C_{v}$ is the specific heat and the value is $718 \mathrm{~J} /(\mathrm{kg} \cdot \mathrm{K})$. At the same time we assume that all the heat energy were used to increase the potential temperature increment. $\bar{\rho}$ is mean atmospheric density. $V_{0}$ presents the volume of the initial cloud and $\Delta \theta$ presents the potential temperature increment of the explosive fireball. Table 3 shows the calculated condition of the explosive tests.

Table 3: Calculated condition of the explosive experiments.

\begin{tabular}{|c|c|c|c|c|c|}
\hline Test & $\begin{array}{c}\text { Mean } \\
\text { temperature } \\
/{ }^{\circ} \mathrm{C}\end{array}$ & $\begin{array}{c}\text { Mean } \\
\text { velocity } \\
/\left(\mathrm{m}^{-1} \mathrm{~s}^{-1}\right.\end{array}$ & $\begin{array}{c}\text { Brunt-Vaisala } \\
\text { frequency } \\
/ \mathrm{s}^{-2}\end{array}$ & $\begin{array}{c}\text { Potential } \\
\text { temperature } \\
\text { increment } \\
/ \mathrm{K}\end{array}$ & $\begin{array}{c}\text { Initial } \\
\text { cloud } \\
\text { radius } \\
/ \mathrm{m}\end{array}$ \\
\hline $\mathrm{BZ4}$ & 24.906 & 1.5264 & $1.648 \times 10^{-3}$ & 47.02 & 0.711 \\
\hline $\mathrm{BZ6}$ & 26.756 & 0.7861 & $2.136 \times 10^{-3}$ & 46.89 & 1.11 \\
\hline $\mathrm{BZ8}$ & 29.266 & 2.8195 & $-1.36 \times 10^{-4}$ & 46.749 & 2.06 \\
\hline $\mathrm{BZ12}$ & 28.482 & 1.5631 & $7.17 \times 10^{-4}$ & 47.063 & 2.465 \\
\hline $\mathrm{BZ18}$ & 25.403 & 0.964 & $2.23 \times 10^{-3}$ & 47.308 & 3.75 \\
\hline $\mathrm{BZ19}$ & 25.403 & 0.9638 & $1.65 \times 10^{-3}$ & 47.296 & 2.297 \\
\hline
\end{tabular}

The "Mean temperature", "Mean velocity" and "Brunt-Vaisala frequency" represent the mean temperature, mean velocity and the Brunt-Vaisala frequency of the atmospheric environment respectively during the tests. "Potential temperature increment" represents the value of the potential temperature increment calculated from equation (6). "Initial cloud radius" represents the radius of the cloud measuring from CCD camera when the fireball crush out. 


\subsection{Potential temperature increment of deflagrable experiment}

Due to the character of the deflagrable test, the deflagrable energy cannot be exacted since we cannot make sure how much fuel is joined to the deflagration. We adopt the infrared photography to determinate the temperature of the fireball. We can obtain the potential temperature increment from the temperature increment. The calculated parameters are shown in Table 4.

Table 4: Calculated condition of deflagrable experiment.

\begin{tabular}{|c|c|c|c|c|c|}
\hline Test & $\begin{array}{c}\text { Mean } \\
\text { temperature } \\
/{ }^{\circ} \mathrm{C}\end{array}$ & $\begin{array}{c}\text { Mean } \\
\text { velocity/ } \\
\mathrm{m} \cdot \mathrm{s}^{-1}\end{array}$ & $\begin{array}{c}\text { Brunt-Vaisala } \\
\text { frequency/s }\end{array}$ & $\begin{array}{c}\text { Potential } \\
\text { temperature } \\
\text { increment } \\
/ \mathrm{K}\end{array}$ & $\begin{array}{c}\text { Initial } \\
\text { cloud } \\
\text { radius } \\
/ \mathrm{m}\end{array}$ \\
\hline BR1 & 25.928 & 1.461 & $1.406 \times 10^{-3}$ & & \\
\hline BR2 & 26.303 & 1.523 & $1.394 \times 10^{-3}$ & 324.4 & 4.594 \\
\hline BR3 & 26.526 & 1.111 & $1.484 \times 10^{-3}$ & & \\
\hline BR4 & 26.655 & 1.294 & $1.396 \times 10^{-3}$ & 324.2 & 4.336 \\
\hline BR5 & 27.034 & 1.395 & $1.189 \times 10^{-3}$ & 324.1 & 4.116 \\
\hline BR6 & 27.034 & 1.395 & $1.189 \times 10^{-3}$ & 323.6 & 2.857 \\
\hline BR7 & 26.665 & 2.473 & $1.755 \times 10^{-3}$ & 324.0 & 2.735 \\
\hline
\end{tabular}

The "Potential temperature increment" represents the value of the potential temperature increment resulting from temperature increment of the fireball. "Initial cloud radius" represents the radius of the cloud measuring from CCD camera when the fireball crush out. Other parameters are the same as mentioned above.

\section{Results and discussion}

We use the uniform grid of $0.4 \mathrm{~m}$ and suppose the underside of the thermal were placed above the bottom $1.5 \mathrm{~m}$. The bottom of the calculated space is rigid wall, and the other sides are freedom .Figure 6 shows the comparison of the cloud top altitudes and radius of the explosive experiment and calculated results. Figure 7 represents the comparison of the cloud top altitudes and radius of the deflagrable experiment and calculated results. Where the "top_comp" and the "rad_comp" represent the calculated results of the cloud top altitude and radius respectively; and the "top_experiment" and the "rad_experiment" indicate the observed results of the top altitude and radius respectively. 


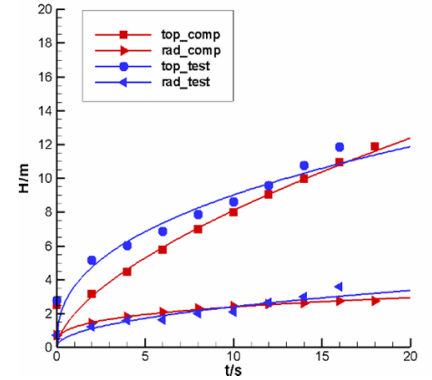

(a) Explosive experiment of BZ4

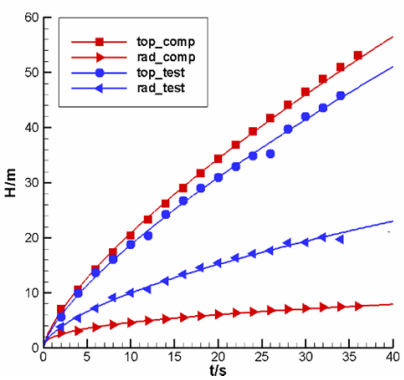

(b) Explosive experiment of BZ19

Figure 6: Comparison of the explosive test and calculated results of top altitudes and radii.

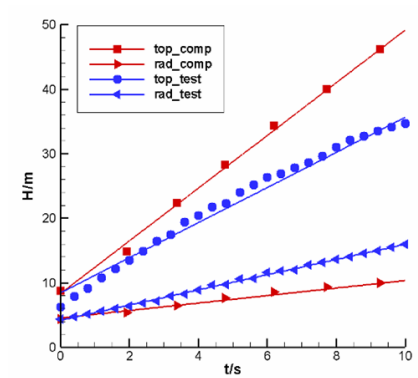

(a) Deflagrable experiment of BR4

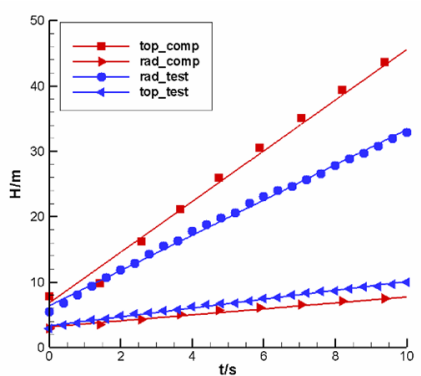

(b) Deflagrable experiment of BR6

Figure 7: Comparison of the deflagrable test and calculated results of top altitudes and radii.

From the results, we can see that the tendency of the calculated curve closed to the results of the experiments. And the variation rates of the top altitude of the cloud are greater than the variation rate of the radius to both the numerical calculated results and the experiment results. But some differences are existing:

a. Top altitudes of numerical results are larger than that of experiments. At the same time, radii of the numerical results are smaller than experiments.

b. Top altitudes and radii of the explosive clouds become slow with time while deflagrable clouds appear to be linear with time.

We give some explanations:

To the first difference, we use a package containing carbonic powder placed on an explosive stuff, which make us difficult to determine the radius of the initial cloud. And the difference of the initial value can result different simulated value. Besides, more and more circumambient matter is involved into the clouds with the rise of the cloud. The effect of the atmospheric turbulence can also decrease the altitude of the explosive cloud and increase the radius of the cloud. At last, the fireball of the deflagrable experiment looks more like an ellipsoid thermal. The transverse radius is greater than the lengthwise radius of the fireball. We use 
the maximal fireball radius as the spherical thermal in the calculation. This reason can also decrease the top altitude of the cloud and increase the radius of the cloud.

To the second difference, the value of the potential temperature increment is decreased and which result in reducing of the buoyancy with the movement of the cloud. The top altitude and the radius of the explosive cloud become slow with time. To the deflagrable experiment, results seem linear with time because short record time owing to large radius of cloud and small pantoscopic of camera.

\section{Conclusions}

We present an experimental approach to study the movement of the explosive and deflagrable cloud. We measure both the fireball and the cloud at the same time rather than the cloud as the former. Based on the measured fireball parameter and the assumption that the main effect on the cloud rising is gravity and buoyancy, we provide a numerical model on the movement of the thermal considering the meteorological condition and have a comparison to data of field experiments. The experimental method and the numerical model are reasonable and can be studied further.

\section{Acknowledgements}

We would like to thank Dr Li L. and Dr Ren X. of Research Institute of Chemical Defense, who assisted us to do the experiment. We also would like to thank Dr Gao Z. and Dr An J. of Institute of Atmospheric Physics of National Academy of Science for providing the support of the weather observation.

\section{References}

[1] Taylor, G. I., Dynamics o f a mass of hot gas rising in air. LA Report 236. 1945.

[2] Morton, B. R., Taylor, G. I. \& Turner, J. S., Turbulent gravitational convection from maintained and instantaneous source. Proc Roy Soc, 1956, A234: 1-23.

[3] Zaslavskii, B. I. \& Sconikov, I. M., Experimental study on buoyant vortex rings. J Applied Mech and Tech Phys, 1983, 24: 16-21.

[4] Zheng, Y., On the instantaneous heat source (explosion clouds) buoyant vortex rings. Beijing: Univ. of Tsinghua, 2008.

[5] Thielen, H. \& Schrodl, E., Blast experiment for the derivation of initial cloud dimension after a "Dirty Bomb" event. Eurosafe Forum 2004. http://www.eurosafe-forum.org/files/pe_103_24_1_3_10_blast_experiments dirty_bomb_thielen_041109.

[6] Makhviladze, G. M., Roberts, J. P. \& Yakush, S. E., Modelling of atmospheric pollution by explosions. Environmental Software, 1995, 10(2): 117-127.

[7] Krispin, J. \& Collins, J. P., Simulations of dusty flows in an incompressible gas using the projection method. AIAA 95-2164.

[8] Brown, P., Krispin, J. \& Potts, M. A., Projection methods for incompressible multiphase cloud-rise phenomena. AIAA 99-3321. 